\title{
Aortic and cardiac air emboli in an older woman
}

\author{
Melody Ren MD, Anna Zavodni MD
}

Cite as: CMAJ 2018 February 5;190:E137-8. doi: 10.1503/cmaj.170733

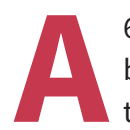

68-year-old woman underwent a transthoracic lung biopsy of a pulmonary nodule guided by computed tomography (CT), suspected to be a metastatic urothelial carcinoma. The procedure was performed with the patient lying in the prone position. A 19-gauge guiding cannula was uneventfully advanced to the leading edge of the nodule in the left lower lobe and was sampled coaxially in five passes using a 20-gauge core needle and a Bard Magnum biopsy instrument (22 $\mathrm{mm}$ throw). Immediately after the biopsy samples were obtained, the patient described severe chest pain and an inability to move her right arm. The biopsy needle was promptly removed. We suspected air embolism and immediately repeated the thoracic CT scan; the scan showed air within the left ventricle and aorta (Figure 1). We consulted the hospital critical care and cardiology teams. The patient was given oxygen and instructed to lie still on the CT gantry until she was helped by staff into the right lateral decubitus position. Repeat CT imaging of the thorax was performed every 30 minutes, showing complete resolution of the air emboli coinciding with symptom resolution after four hours. Troponin levels peaked at $67 \mathrm{ng} / \mathrm{L}$; an electrocardiogram and an echocardiogram were unremarkable. The patient was admitted to the internal medicine service for further monitoring. Magnetic resonance imaging of the brain 19 hours after the initial biopsy showed no acute intracranial events. The patient remained stable during the admission and was discharged 24 hours after being admitted to the internal medicince service.

\section{Discussion}

Systemic air emboli are an uncommon ( $0.02 \%$ to $0.4 \%)$ complication of CT-guided lung biopsies. ${ }^{1-4}$ Other common causes of air emboli include surgery (especially neurosurgical and otolaryngological procedures), trauma, catheterization and other intravascular interventions, and barotrauma from mechanical ventilation and diving. ${ }^{5}$ Air emboli occur when a direct channel of communication forms between a vessel and source of air and the pressure gradient favours air being pulled into the vessel.,5 This situation can occur during CT-guided lung biopsy when the introducer is left to open air, when the needle creates a fistula between bronchi and the vasculature along its trajectory, or when repetitive aspirations required to obtain the biopsy sample inadvertently inject air into the vessel.

Typically, arterial air emboli have more substantial consequences than venous emboli. The pulmonary vasculature can

\section{KEY POINTS}

- Systemic air emboli, both arterial and venous, are an uncommon complication of lung biopsy guided by computed tomography (CT).

- Direct communication between the vasculature and air source with a pressure gradient favouring air entry into the vessel is required to form air emboli.

- Air emboli should be suspected when a patient has manifestations of rapid cardiac, respiratory, neurologic or hemodynamic deterioration during or soon after CT-guided lung biopsy; confirmatory imaging is needed to differentiate air emboli from other causes of acute decompensation.

- Initial management of air emboli is to stabilize the patient with resuscitative measures; specific management includes giving $100 \%$ oxygen, positioning the patient to prevent further movement of the emboli and considering treatment with hyperbaric oxygen.

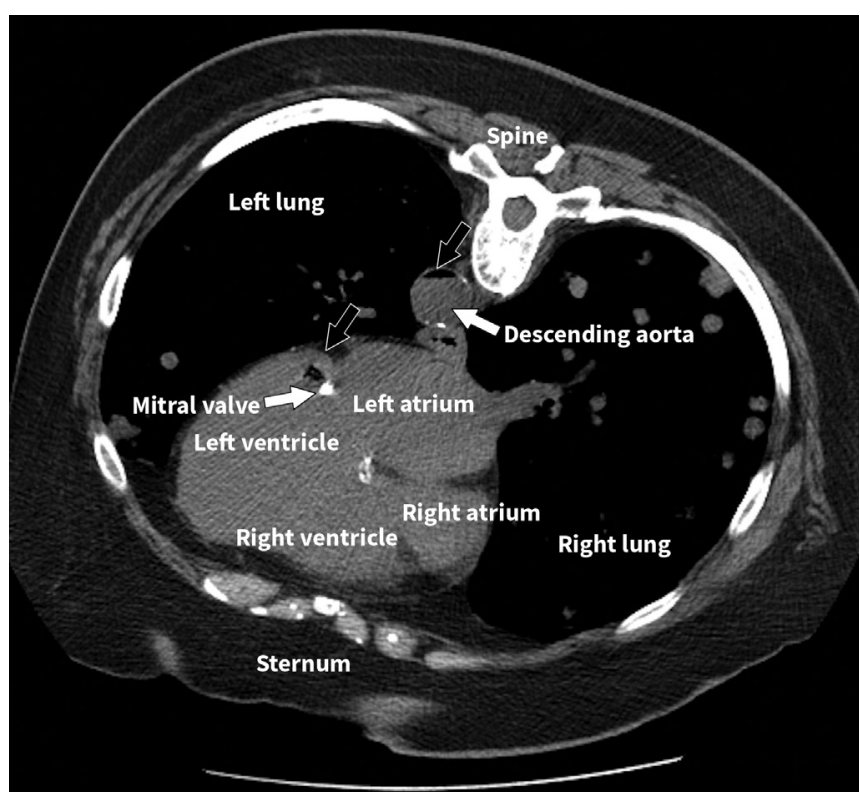

Figure 1: Computed tomography (CT) of the thorax of a 68-year-old woman who reported chest pain and an inability to move her right arm immediately after undergoing a CT-guided transthoracic lung biopsy of a pulmonary nodule. The patient was in the prone position so that the lesion in the posterior left lower lobe could be accessed; the left side of the image shows the patient's left side. The black arrows show air in the descending aorta (top) and left ventricle (bottom) adjacent to the calcified mitral valve. Multiple nodules can be seen in the lungs on both the right and left sides. 
remove venous gas by diffusion into the alveoli. Research to test the maximum volume able to be diffused by the pulmonary vasculature has been done using animal models; the upper limit before the filtering capacity of the lungs is exceeded has been reported to be $30 \mathrm{~mL}$ in canines and a rate of $10.5 \mathrm{~mL} / \mathrm{min}$ of air for a $70-\mathrm{kg}$ human, extrapolated from studies of sheep. ${ }^{7} \mathrm{~A}$ bolus of 200-300 mL of air in the venous system can be fatal because of obstructive shock and the associated inflammatory response. ${ }^{8}$ Venous air emboli can become arterial when the lung's capacity to filter out gas is exceeded or in the presence of a patent foramen ovale or septal defect. Arterial air emboli may cause obstructive ischemic end-organ damage, particularly in the cerebral and coronary systems, because of the heightened vulnerability of these systems to hypoxia. ${ }^{6}$

The volume of air has less impact on clinical outcomes than the location of the embolism; any amount of air in an important vessel can have important consequences. Presenting symptoms can overlap with those of acute coronary syndromes, stroke and other thromboembolic events; however, emerging data suggest that radiologic evidence of air emboli after $\mathrm{CT}$-guided lung biopsy does not necessarily correlate with symptoms. ${ }^{2}$

When a patient has manifestations of rapid cardiac, respiratory, neurologic or hemodynamic deterioration during or soon after CT-guided lung biopsy, air embolism should be considered in the differential diagnosis. Management should be tailored to the patient's medical stability and specific presentation. The biopsy needle should be promptly removed and initial resuscitation should be conducted with the cardiac arrest team, including starting mechanical ventilation, intravenous fluids and vasopressors as necessary. Once the patient's condition is stable, repeat imaging of the brain and thorax should be considered to confirm the diagnosis, as well as other bloodwork and investigations targeted at the presenting symptoms, such as an electrocardiography and echocardiography. The specific management of air emboli includes placing patients on $100 \%$ high-flow oxygen to increase the nitrogen gradient between the blood and the air emboli and accelerate air resorption. ${ }^{6}$

\section{Prevention and management of cerebral embolization}

Patients should be positioned according to the type of embolism to prevent cerebral embolization. ${ }^{2,6}$ For venous air emboli, patients should be placed in the left lateral decubitus position and the Trendelenburg position (with the feet 15-30 degrees higher than the head) to trap the air in the right ventricle above the right heart outflow tract. For arterial air emboli, both the supine and the right lateral decubitus and Trendelenburg positions are suggested. Advocates of the right lateral decubitus and Trendelenburg positions suggest they allow for air to be trapped in the left ventricle above the left heart outflow tract. ${ }^{6}$ In addition, many CT-guided lung biopsies are performed with the patient in the prone position, and turning a patient from the prone to the supine position can allow for movement of air emboli. ${ }^{9}$ Others suggest that forceful arterial blood flow will negate attempts to trap the air in the most nondependent location in the heart, and the supine position can help to reduce cerebral edema from cerebral air embolism. ${ }^{5}$ To our knowledge there is no evidence supporting one method over the other. The patient should remain in position for sufficient time to allow for resorption as confirmed by symptoms and imaging.

Air within the brain warrants consultation with neurosurgery. Small, stable intracardiac air bubbles can be monitored with repeated imaging at intervals while the patient remains immobilized on the CT gantry.

If there are neurological deficits or end-organ damage, treatment with hyperbaric oxygen should be considered as the definitive therapy for arterial air emboli, ideally within four to six hours after symptoms onset. ${ }^{10}$ The benefits of this therapy should be weighed against the potential risk of transporting a sick patient to a centre that offers hyperbaric oxygen. An absolute contraindication to treatment with hyperbaric oxygen is an untreated pneumothorax. ${ }^{5}$ Other therapies for systemic air emboli that do not have definitive evidence of benefit in humans at this time include anticoagulation, steroids, lidocaine, manual air aspiration and chest compressions.

Systemic air emboli frequently lead to poor outcomes, with estimates derived from case reports suggesting that almost all symptomatic air emboli result in cardiac and/or cerebral morbidity and often death. ${ }^{2}$ However, prompt recognition and management can improve outcomes. ${ }^{1}$

\section{References}

1. Richardson CM, Pointon K, Manhire A, et al. Percutaneous lung biopsies: a survey of UK practice based on 5444 biopsies. Br J Radiol 2002;75:731-5.

2. Hiraki T, Fujiwara H, Sakurai J, et al. Nonfatal systemic air embolism complicating percutaneous CT-guided transthoracic needle biopsy. Chest 2007;132:684-90.

3. Sinner WN. Complications of percutaneous transthoracic needle aspiration biopsy. Acta Radiol Diagn (Stockh) 1976;17:813-28.

4. Tomiyama N, Yasuhara Y, Nakajima Y, et al. CT-guided needle biopsy of lung lesions: a survey of severe complication based on 9783 biopsies in Japan. Eur $J$ Radiol 2006;59:60-4.

5. Muth CM, Shank E. Gas embolism. N Engl J Med 2000;342:476-82.

6. Hare SS, Gupta A, Goncalves A, et al. Systemic arterial air embolism after percutaneous lung biopsy. Clin Radiol 2011;66:589-96.

7. Murphy BP, Harford F, Cramer F. Cerebral air embolism resulting from invasive medical procedures treatment with hyperbaric oxygen. Ann Surg 1985;201:242-5.

8. Toung TJ, Rossberg M, Hutchins G. Volume of air in a lethal venous air embolism. Anesthesiology 2001;94:360-1.

9. Bhatia S. Systemic air embolism following CT-guided lung biopsy. J Vasc Interv Radiol 2009;20:709-11.

10. Blanc $P$, Boussuges $A$, Henriette $K$, et al. latrogenic cerebral air embolism: importance of an early hyperbaric oxygenation. Intensive Care Med 2002;28:559-63.
Competing interests: None declared.

This article has been peer reviewed.

The authors have obtained patient consent.

Affiliation: Department of Medicine, University of Toronto, Toronto, Ont.
Contributors: Both authors contributed to the conception and design of the work, drafted the manuscript, revised it critically for important intellectual content, gave final approval of the version to be published and agreed to be accountable for all aspects of the work.

Correspondence to: Melody Ren, melody.ren@medportal.ca 